\title{
Increased plasma levels of CK-18 as potential cell death biomarker in patients with HELLP syndrome
}

\author{
K John ${ }^{1}$, S Wielgosz ${ }^{1}$, K Schulze-Osthoff ${ }^{2,3}$, H Bantel ${ }^{*, 1,5}$ and R Hass ${ }^{4,5}$
}

HELLP (hemolysis, elevated liver enzymes, low platelets) syndrome represents a life-threatening pregnancy disorder with high fetal and maternal mortality, but its underlying molecular mechanisms remain unknown. Although apoptosis has been implicated in HELLP syndrome, its pathogenic role remains largely unclear. In the present study, we investigated whether the detection of apoptosis by novel plasma biomarkers is of diagnostic value in HELLP patients. For this purpose, we analyzed two biomarkers that specifically detect apoptosis or overall cell death of epithelial cells, such as hepatocytes or placental trophoblasts, through the release of caspase-cleaved or total (caspase-cleaved and uncleaved) cytokeratin-18 (CK-18) in plasma of HELLP patients compared with pregnant as well as non-pregnant healthy women. In addition, caspase activation and cell death were determined in placental tissues of HELLP patients and individuals with normal pregnancy. In contrast to pregnant or non-pregnant healthy controls, we observed significantly increased levels of both caspase-cleaved and total CK-18 in plasma of HELLP patients. Following delivery, CK-18 levels rapidly decreased in HELLP patients. Caspase activation and cell death were also elevated in placental tissues from HELLP patients compared with healthy pregnant women. These data demonstrate not only that apoptosis is increased in HELLP syndrome, but also that caspase-cleaved or total CK-18 are promising plasma biomarkers to identify patients with HELLP syndrome. Thus, further studies are warranted to evaluate the utility of these biomarkers for monitoring disease activity in HELLP syndrome.

Cell Death and Disease (2013) 4, e886; doi:10.1038/cddis.2013.408; published online 24 October 2013

Subject Category: Experimental Medicine

HELLP (hemolysis, elevated liver enzymes, low platelets) syndrome and pre-eclampsia are multisystemic diseases associated with high perinatal and maternal morbidity and mortality. ${ }^{1,2}$ Both entities are considered to represent different severity of the same pregnancy disorder and thus show similar pathological alterations of the placenta. ${ }^{3}$ Although the causes of this pregnancy disorder still remain unclear, a variety of findings points to endothelial dysfunctions as one of the early events in this multistep disease, whereby increased levels of the anti-angiogenic factor sFlt-1 (soluble fms-like tyrosine kinase-1) coincide with reduced vascularization and an aberrant placentation. These effects are associated with increased vasoconstriction, maternal hypertension and reduced uteroplacental blood flow. As a consequence, an accumulation of oxidative stress-induced metabolites such as reactive oxygen species triggers placental tissue damage. ${ }^{4-6}$ Such placental tissue damage attracts and activates immunecompetent cells. Indeed, immunological maladaptation is the most probable trigger of the insult to the invading trophoblast, which also occurs early in the first trimester. This could result in a sustained activation of the immune system, ${ }^{7,8}$ which imbalances metabolic pathways and interferes with important regulatory mechanisms such as apoptosis. In this context, normal placenta development is controlled by physiological apoptosis, which has a crucial role in the maintenance of tissue homeostasis and an intact pregnancy. ${ }^{9,10}$ In pregnancies, complicated by pre-eclampsia increased apoptosis of placental tissue compared with normal pregnancy has been demonstrated. ${ }^{11-14}$

Apoptosis is triggered by two major signaling routes, namely the extrinsic death receptor and the intrinsic mitochondrial pathway. ${ }^{15-17}$ Binding of death ligands, such as tumor necrosis factor- $\alpha$, tumor necrosis factor-related apoptosis-inducing ligand or CD95L, to their respective death receptors leads to death-inducing signaling complex formation, which results in receptor oligomerization and activation of initiator caspase-8 and -10. Subsequently, initiator caspases activate effector caspases such as caspase-3 and -7 . The intrinsic pathway is triggered by cellular stress, which initiates the release of mitochondrial proapoptotic mediators followed by activation of initiator caspase- 9 and downstream effector caspases. Effector caspases cleave a variety of cellular

\footnotetext{
${ }^{1}$ Department of Gastroenterology, Hepatology and Endocrinology, Hannover Medical School, Hannover, Germany; ${ }^{2}$ Department of Molecular Medicine, Interfaculty Institute for Biochemistry, University of Tübingen, Tübingen, Germany; ${ }^{3}$ German Cancer Consortium (DKTK) and German Cancer Research Center, Heidelberg, Germany and ${ }^{4}$ Biochemistry and Tumor Biology Laboratory, Gynecology Research Unit, Department of Obstetrics and Gynecology, Hannover Medical School, Hannover, Germany

${ }^{*}$ Corresponding author: H Bantel, Department of Gastroenterology, Hepatology and Endocrinology, Hannover Medical School, Carl-Neuberg-Strasse 1, Hannover 30625, Germany. Tel: +49 511 5329514; Fax: +49 511-5326998; E-mail: Bantel.Heike@mh-hannover.de

${ }^{5}$ These authors contributed equally.

Keywords: cytokeratin-18; apoptosis; caspases; HELLP syndrome; pregnancy disorder; biomarker

Abbreviations: ALT, alanine aminotransferase; AST, aspartate aminotransferase; CK-18, cytokeratin-18; HELLP, hemolysis, elevated liver enzymes, low platelets; LDH, lactate dehydrogenase; TUNEL, terminal deoxynucleotidyl transferase dUTP nick end labelling

Received 24.8.13; revised 14.9.13; accepted 16.9.13; Edited by H-U Simon
} 
substrates resulting in the demise of the cell. Among the different substrates of caspases are proteins with diverse biological functions including structural proteins of the cytokeratin I family such as cytokeratin-18 (CK-18).

CK-18 is specifically expressed by epithelial cells, such as hepatocytes or placental trophoblasts. ${ }^{18,19}$ It has been recently demonstrated that caspase-generated CK-18 cleavage fragments are released from apoptotic epithelial cells and can be detected in blood samples from patients with epithelial organ dysfunction, for example, with liver diseases or pre-eclampsia. ${ }^{20-23}$ The molecular mechanisms of CK-18 release from apoptotic epithelial cells remain unknown. During apoptosis, caspase-generated CK-18 fragments have been found in cytoplasmic inclusion bodies that are brought to apoptotic surface blebs, most likely before secondary necrosis occurs. ${ }^{24}$

In the present study, we investigated different cell death biomarkers including caspase-cleaved CK-18 and total (caspase-cleaved and uncleaved) CK-18 in plasma samples from patients with HELLP syndrome compared with individuals with normal pregnancy. We found significantly higher plasma levels of caspase-cleaved and total CK-18 in HELLP syndrome compared with pregnant or non-pregnant healthy controls. Our results suggest that cell death biomarkers might therefore help to early identify patients with increased risk of developing life-threatening HELLP syndrome.

\section{Results}

Increased CK-18 plasma levels in patients with HELLP syndrome compared with normal pregnancy. During apoptosis of epithelial cells, for example, hepatocytes or trophoblasts, caspases cleave the intermediate filament protein CK-18 into fragments that are released in the bloodstream. CK-18 cleavage thereby generates a neoepitope that can be detected by the monoclonal antibody M30 and therefore allows the assessment of apoptosis specifically of epithelial cells by an ELISA. In contrast, another assay, the M65 ELISA, detects both caspasecleaved and uncleaved $\mathrm{CK}-18$ and is therefore used as a marker of overall death including apoptosis and necrosis.

To investigate the utility of both cell death biomarkers, we analyzed plasma samples of HELLP patients $(n=15)$ compared with individuals with normal pregnancy $(n=20)$. We found significantly $(P<0.01)$ higher plasma levels of CK-18 fragments in HELLP patients compared with women with normal pregnancy $(504.0 \pm 93.5 \mathrm{U} / \mathrm{l}$ versus $203.9 \pm 15.4$ $\mathrm{U} / \mathrm{l}$; Figure 1a). HELLP patients showed also significantly $(P<0.01)$ higher $\mathrm{CK}-18$ fragment levels compared with nonpregnant healthy women $(218.0 \pm 18.1 \mathrm{U} / / ; n=10)$, whereas individuals with normal pregnancy did not differ from nonpregnant controls in their plasma levels of CK-18 fragments (Figure 1a). Similar results were obtained for the detection of total CK-18 with significantly $(P<0.01)$ higher $\mathrm{CK}-18$ levels in HELLP patients $(2633.8 \pm 595.7 \mathrm{U} / \mathrm{l})$ compared with women with normal pregnancy $(652.8 \pm 30.4 \mathrm{U} / \mathrm{l})$ and non-pregnant healthy controls (261.3 $\pm 33.6 \mathrm{U} / \mathrm{l}$; Figure $1 \mathrm{~b})$.

CK-18 cell death biomarkers reveal a high sensitivity and specificity for the discrimination between HELLP and normal pregnancy. To determine the predictive discriminating values of caspase-cleaved CK-18 and total CK-18 for discrimination between patients with HELLP syndrome $(n=15)$ and women with normal pregnancy $(n=20)$, we performed a receiver operating characteristics analysis comparing patients with HELLP syndrome and women with normal pregnancy (Figure 2). Caspase-cleaved CK-18 levels above or below $243 \mathrm{U} / \mathrm{l}$ correctly predicted HELLP syndrome with a sensitivity of $87 \%$ and a specificity of $80 \%$ (area under the curve $=0.89$; Figure 3a). For total (caspase-cleaved and uncleaved) CK-18 levels, we calculated a cutoff value of $1032 \mathrm{U} / \mathrm{l}$ for the prediction of HELLP syndrome with a sensitivity and a specificity of $100 \%$ (area under the curve=1.0; Figure 3b).

No correlation between CK-18 fragment and aminotransferase levels in patients with HELLP syndrome. To analyze whether increased CK-18 fragment levels are associated with liver damage, we performed regression analyses correlating $\mathrm{CK}-18$ fragments with aminotransferase and lactate dehydrogenase (LDH) levels of HELLP syndrome patients. We found no positive correlation of caspase-mediated CK-18 cleavage fragments with aspartate aminotransferase (AST) or alanine aminotransferase (ALT) levels ( $r=-0.48$ and $r=-0.23$; Figures $3 a$ and $b$ ), indicating that CK-18 fragments and aminotransferases are released by different mechanisms or that $\mathrm{CK}-18$ fragment
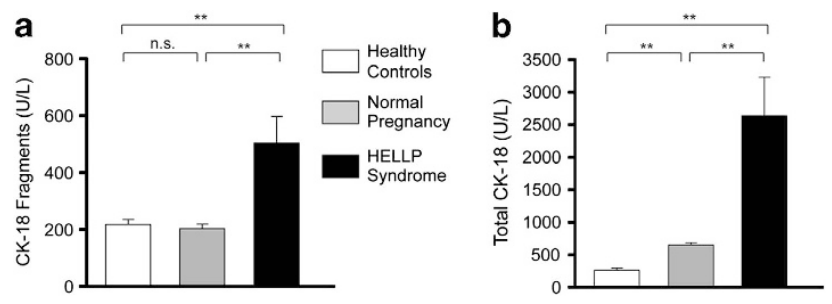

Figure 1 Detection of caspase-cleaved CK-18 fragments (a) and total CK-18 (b) in plasma samples of patients with HELLP syndrome $(n=15)$, women with normal pregnancy $(n=20)$ and non-pregnant healthy women $(n=10)$. Patients with HELLP syndrome showed significantly higher plasma levels of CK-18 fragments (a) and total CK-18 (b) compared with women with normal or without pregnancy. ${ }^{*} P<0.01 ;$ n.s., non significant
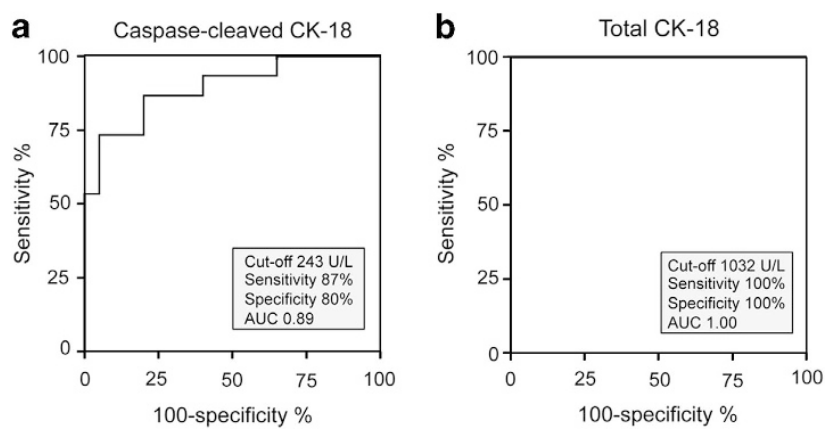

Figure 2 Prediction of HELLP syndrome by cell death biomarkers. The receiver operating characteristic analyses indicate the threshold for caspase-cleaved (a) and total (b) CK-18 with the best compromise sensitivity/specificity for discrimination between HELLP syndrome and normal pregnancy. AUC, area under the curve 
levels are not related to liver damage. In contrast, a positive correlation of CK-18 fragments (Figure $3 \mathrm{c}$ ) or total CK-18 (Figure 3d) was found with LDH levels ( $r=0.27$ and $r=0.42$ ). Moreover, CK-18 fragments showed an inverse correlation with platelet counts $(r=-0.29$, data not shown), further suggesting that CK-18 cell death biomarkers might be associated with non-hepatic rather than with hepatic disease activity.
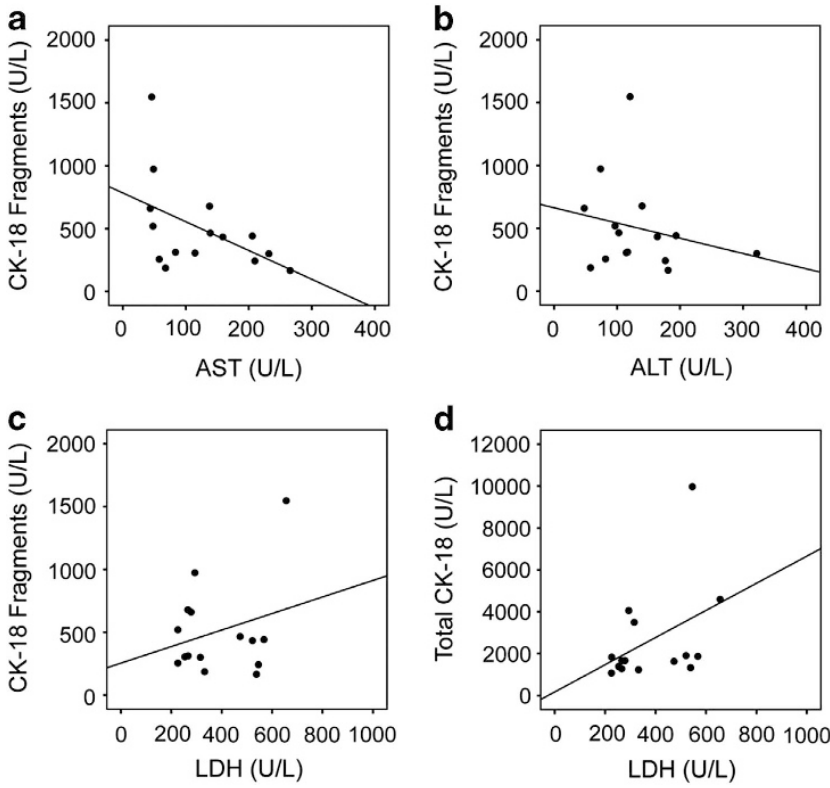

Figure 3 Regression analyses correlating CK-18 fragments with AST (a), ALT (b) or LDH (c) levels as well as total CK-18 with LDH levels (d) in HELLP syndrome patients. No positive correlation between CK-18 fragments and aminotransferase levels was found. In contrast, CK-18 fragments and total CK-18 positively correlated with LDH levels
Increased caspase activation and cell death in placenta tissue of patients with HELLP syndrome compared with individuals with normal pregnancy. One reason for the lack of correlation between CK-18 fragments and aminotransferase levels might be that the detected CK-18 fragments are mainly released from placenta tissues in HELLP patients. We therefore investigated caspase activation and cell death in placenta tissues of HELLP syndrome patients $(n=5)$ compared with normal pregnant women $(n=4)$. As assessed by immunostaining with an antibody against active caspase-3, we found a higher number of cells positive for active caspase-3 in placentas of HELLP syndrome patients compared with normal pregnancy (Figure 4a). Similarly, terminal deoxynucleotidyl transferase dUTP nick end labelling (TUNEL) staining showed that patients with HELLP syndrome revealed a significantly $(P<0.05)$ higher cell death rate compared with healthy pregnancy $(39.8 \pm 8.6 \%$ versus $7.4 \pm 4.1 \%)$, indicating elevated placental cell death in those patients compared with normal pregnant women (Figures $4 b$ and $c$ ).

Decrease of CK-18 plasma levels in HELLP syndrome patients after delivery. We then analyzed the course of CK-18 plasma levels one day before and after delivery of HELLP syndrome patients $(n=3)$. Both caspase-mediated CK-18 fragments (Figure $5 \mathrm{a}$ ) as well as total CK-18 (Figure 5b) levels decreased after delivery (450.2 \pm 105.2 $\mathrm{U} / \mathrm{l}$ and $978.9 \pm 89.7 \mathrm{U} / \mathrm{l})$ compared with the levels before delivery $(918.9 \pm 383.2 \mathrm{U} / \mathrm{l}$ and $2645.0 \pm 1026.7 \mathrm{U} / \mathrm{I})$. These data indicate that detection of epithelial cell death in blood samples might represent reliable noninvasive biomarkers for monitoring disease activity in HELLP syndrome.

\section{Discussion}

HELLP syndrome displays a life-threatening pregnancy disorder without reliable early diagnostic biomarkers so far

a
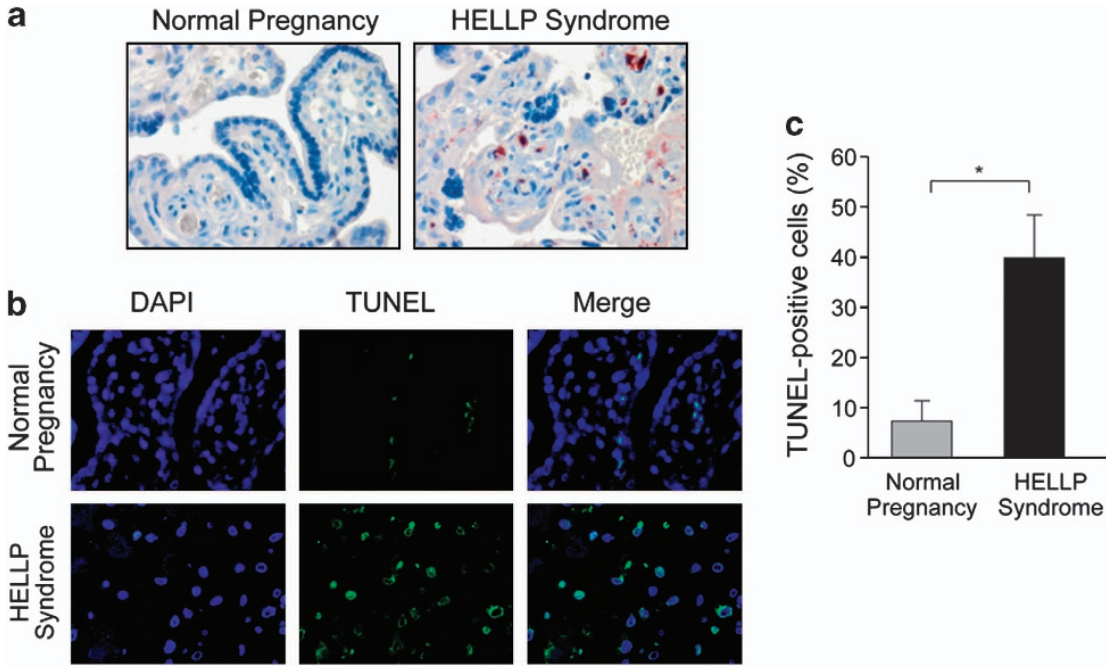

Figure 4 Detection of caspase-3 activation (a) and TUNEL reactivity $(\mathbf{b})$ in placenta tissues of patients with HELLP syndrome $(n=5)$ and women with normal pregnancy $(n=4)$. The percentage of TUNEL-positive cells was assessed by analyzing four microscopic fields at $\times 400$ magnification and is given as mean \pm S.E.M. (c). Patients with HELLP syndrome showed increased caspase-3 activation and a higher percentage of TUNEL-positive cells in placenta tissues compared with normal pregnant women. ${ }^{*} P<0$.05. DAPI, 4',6-diamidino-2-phenylindole 

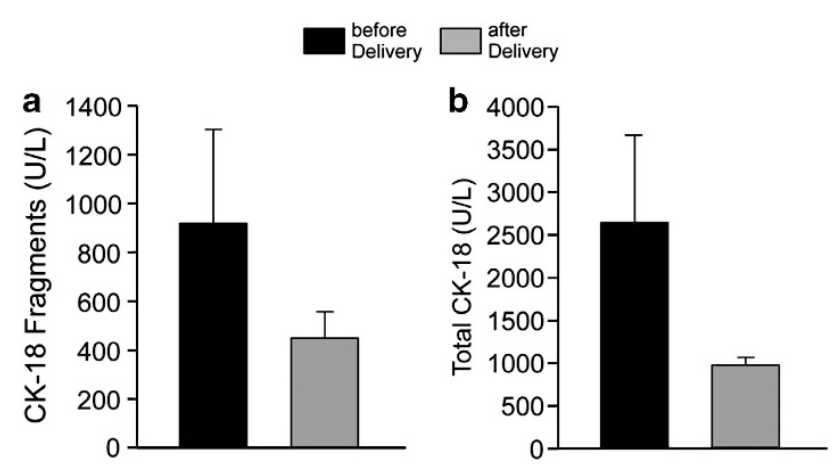

Figure 5 Detection of caspase-cleaved CK-18 fragments (a) and total CK-18 (b) in plasma samples of patients with HELLP syndrome $(n=3)$ within one day before and after delivery. Both cell death biomarkers rapidly declined after delivery

and delivery by an emergency case Cesarean section has to be performed in a large number of cases. Apoptosis has been implicated in HELLP syndrome, although its pathogenic contribution to placental and liver damage in this disease remains unknown. ${ }^{9,25}$ There is increasing evidence that caspase-cleaved CK-18 and total CK-18 represent useful noninvasive biomarkers for serological detection of epithelial organ damage. ${ }^{20-23}$ In the present study, we therefore analyzed those cell death biomarkers in plasma of women with normal pregnancy or HELLP syndrome.

We could demonstrate significantly elevated plasma levels of CK-18 fragments and total CK-18 in HELLP syndrome compared with normal pregnancy or healthy controls. Both CK-18 biomarkers revealed a high sensitivity and specificity for discrimination between HELLP and normal pregnancy. Whether the obviously better diagnostic performance of the M65 biomarker reflects different plasma stabilities of the various CK-18 forms or differential contribution of apoptosis or necrosis is currently unclear. Interestingly, the elevated CK-18 plasma levels rapidly declined after delivery. In order to evaluate whether increased CK-18 plasma levels are influenced by liver injury, we correlated CK-18 fragments with aminotransferase levels. In this context, we found a negative correlation between caspase-generated CK-18 fragments and aminotransferase levels in HELLP syndrome patients. This observation is in line with a recent study that found no correlation between $\mathrm{CK}-18$ fragments and laboratory markers of liver dysfunction in patients with pre-eclampsia. ${ }^{21}$ CK-18 plasma levels further revealed an inverse correlation with platelet counts (data not shown) and a positive correlation with LDH levels. Thus, these and previous data ${ }^{21}$ would imply that the placenta rather than the liver is the origin of increased plasma CK-18 levels, although further studies are required to address this issue in detail.

The molecular mechanisms of increased cell death in placenta and liver tissues from patients with HELLP syndrome are largely unknown. It has recently been demonstrated that sera from HELLP syndrome patients exert toxicity in primary human hepatocytes and that blocking of CD95L reduced liver toxicity of those sera. In this study, CD95L was found to be produced in the placenta. ${ }^{25}$ In line with this observation, increased CD95L expression and apoptosis of villous trophoblasts of HELLP syndrome compared with pre-eclampsia patients have been demonstrated. ${ }^{26}$ In contrast, several studies reported on reduced CD95L expression but higher apoptosis rates in placenta tissues from patients with pre-eclampsia. ${ }^{12,27}$ We found a higher caspase activity and cell death rate in placenta tissue from patients with HELLP syndrome compared with normal pregnant women. This observation is supported by a recent study demonstrating increased caspase-3 expression and apoptosis in placenta tissues from HELLP syndrome patients compared with normal pregnancy. ${ }^{28}$ Whether the increased caspase activation and apoptosis in placenta tissues of HELLP syndrome patients is mediated by CD95/CD95L interaction remains to be elucidated. CD95L is also physiologically expressed by immune cells such as cytotoxic $T$ lymphocytes. $\mathrm{T}$ cells not only mediate apoptosis of parenchyma cells but also induce T-cell apoptosis via CD95L/CD95-mediated suicide or fratricide, thereby terminating or counter-regulating immune responses. ${ }^{29} \mathrm{~A}$ lack of physiological T-cell death in HELLP syndrome might contribute to increased T-cell-mediated cytotoxicity against placental cells associated with the release of CK-18 fragments. Interestingly, we found lower plasma levels of CD95L in HELLP patients compared with normal pregnancy (data not shown).

Taken together, we have demonstrated that CK-18 cell death biomarkers are detectable at significantly elevated levels in the plasma of HELLP patients and return to levels of healthy individuals after delivery. These noninvasive biomarkers revealed a promising diagnostic performance for the discrimination between HELLP and normal pregnancy and might therefore be suitable for early selection of patients who might benefit from Cesarean section. Further large cohort studies are warranted to evaluate the value of those cell death biomarkers for early detection and monitoring of pregnancy disorders including pre-eclampsia and HELLP syndrome.

\begin{abstract}
Materials and Methods
Patients. We investigated CK-18 cell death biomarkers in plasma samples from 15 patients with HELLP syndrome (mean age $31.0 \pm 1.5$ years; week of gestation mean $31.3 \pm 1.4$ ), 20 women with normal pregnancy and normal aminotransferase levels (mean age $29.8 \pm 5.1$ years; week of gestation mean $38.2 \pm 3.1$ ) and 10 healthy non-pregnant women (mean age $26.1 \pm 1.3$ years). Plasma samples for detection of CK-18 cell death biomarkers were collected within 3 days ( $\leq 1$ day $n=10 ; 2-3$ days $n=5$ ) before delivery in HELLP syndrome patients. Routine laboratory parameters were determined at the same time (mean AST level $=122.1 \pm 19.6 \mathrm{U} / /$; mean ALT level $=130.9 \pm 17.8 \mathrm{U} /$; mean platelet count $127333 \pm 11586 / \mu \mathrm{l}$; mean LDH level $379.5 \pm 38.2 \mathrm{U} /$; m mean hemoglobin level $11.5 \pm 0.3 \mathrm{~g} / \mathrm{dl})$. In three patients with HELLP syndrome, CK-18 cell death biomarkers were measured within one day before and after delivery. In addition, we performed staining for caspase activation and cell death (TUNEL reactivity) in placenta tissues of four women with normal pregnancy and five patients with HELLP syndrome. The study was performed according to the guidelines of the Ethics Committee of Hannover Medical School.
\end{abstract}

Detection of caspase-cleaved and total cytokeratin-18. For the quantitative measurement of the caspase-generated neoepitope of CK-18 in plasma samples, we used the M30-Apoptosense ELISA kit (Peviva, Bromma, Sweden) as described previously. ${ }^{20,22}$ We further used the M65 ELISA (Peviva) that quantifies both uncleaved and caspase-cleaved CK-18. ${ }^{23}$ The assays were performed in duplicates according to the protocol of the manufacturer. ELISA units were calculated based on calibration curves with synthetic immunogenic peptides. For both assays, a cubic spline algorithm was employed for data interpolation.

Immunohistochemistry. Paraffin sections from placenta of HELLP patients and healthy pregnancy were stained for active caspase-3 using an anti-cleaved 
caspase-3 antibody (Cell Signaling Technology, Danvers, MA, USA). The sections were deparaffinized in xylene and ethanol, followed by washing in TBST $(50 \mathrm{mM}$ Tris-Base, $150 \mathrm{mM}$ sodium chloride, $0.05 \%$ Tween-20). Antigen retrieval was performed by cooking in unmasking solution (Vector Laboratories, Burlingame, CA, USA) for $3 \mathrm{~min}$ and nonspecific binding was blocked with $1 \%$ bovine serum albumin for $1 \mathrm{~h}$. Sections were then incubated overnight at $4{ }^{\circ} \mathrm{C}$ with anti-cleaved caspase-3 antibody. After repeated washing, sections were incubated for $30 \mathrm{~min}$ with biotinylated secondary antibody. Following inhibition of endogenous peroxidase, sections were covered for $1 \mathrm{~h}$ with avidin-biotin complex reagent (ABC kit, Vector Laboratories). Finally, the sections were stained in aminoethylcarbazole solution (AEC chromogen kit, Sigma-Aldrich, St. Louis, MO, USA) and counterstained with hematoxylin. For TUNEL staining (cell death detection kit, Roche, Mannheim, Germany), paraffin sections were deparaffinized in xylene and ethanol, followed by washing in PBS and cooking in unmasking solution (Vector Laboratories). Sections were washed in PBS and then incubated for $1 \mathrm{~h}$ at $37^{\circ} \mathrm{C}$ in a reaction mixture containing terminal deoxynucleotidyl transferase and fluorescein isothiocyanate-labeled deoxyuridine triphosphate as described. ${ }^{30}$ Sections were counterstained with 4',6-diamidino-2-phenylindole (Vector Laboratories).

Statistical analyses. Statistical analyses comparing the concentration of the different variables in the plasma of patients and controls were performed by using the Mann-Whitney test (GraphPad Prism 5.0; GraphPad Software, Inc., La Jolla, CA, USA). Regression analyses to calculate the Pearson correlation coefficient and receiver operating characteristics analyses were performed by using SPSS 17.0 (IBM Corporation, Armonk, NY, USA). A P-value of less than 0.05 was considered significant.

\section{Conflict of Interest}

The authors declare no conflict of interest.

Acknowledgements. We thank P. Hillemanns and C. von Kaisenberg for helpful discussions. This study was supported by the BMBF (01FP09104B).

1. Curtin WM, Weinstein L. A review of HELLP syndrome. J Perinatol 1999; 19: 138-143.

2. Goodburn $E$, Campbell O. Reducing maternal mortality in the developing world: sectorwide approaches may be the key. BMJ 2001; 322: 917-920.

3. Smulian J, Shen-Schwarz S, Scorza W, Kinzler W, Vintzileos A. A clinicohistopathologic comparison between HELLP syndrome and severe preeclampsia. J Matern Fetal Neonatal Med 2004; 16: 287-293.

4. McKinney ET, Shouri R, Hunt RS, Ahokas RA, Sibai BM. Plasma urinary, and salivary 8-epi-prostaglandin f2alpha levels in normotensive and preeclamptic pregnancies. Am J Obstet Gynecol 2000; 183: 874-877.

5. Walsh SW, Vaughan JE, Wang Y, Roberts LJ II. Placental isoprostane is significantly increased in preeclampsia. FASEB J 2000; 14: 1289-1296.

6. Hass R, Sohn C. Increased oxidative stress in pre-eclamptic placenta is associated with altered proteasome activity and protein patterns. Placenta 2003; 24: 979-984.

7. Hass R, Staboulidou I, Wüstemann M, Kundu S, Oehler K, Scharf A. Preeclampsia as a multifactorial pregnancy disorder: Clinical symptoms, diagnostic tools and research strategies. Curr Women's Health Rev 2007; 3: 166-176.

8. Abildgaard U, Heimdal K. Pathogenesis of the syndrome of hemolysis, elevated live enzymes, and low platelet count (HELLP): a review. Eur J Obstet Gynecol Reprod Biol 2013; 166: 117-123

9. Straszewski-Chavez SL, Abrahams VM, Mor G. The role of apoptosis in the regulation of trophoblast survival and differentiation during pregnancy. Endocr Rev 2005; 26: 877-897.

10. Abrahams VM, Bole-Aldo P, Kim YM, Straszewski-Chavez SL, Chaiworapongsa T, Romero $\mathrm{R}$ et al. Divergent trophoblast responses to bacterial products mediated by TLRs. J Immunol 2004; 173: 4286-4296.
11. Leung DN, Smith SC, To KF, Sahota DS, Baker PN. Increased placental apoptosis in pregnancies complicated by preeclampsia. Am J Obstet Gynecol 2001; 184: 1249-1250.

12. Allaire AD, Ballenger KA, Wells SR, McMahon MJ, Lessey BA. Placental apoptosis in preeclampsia. Obstet Gynecol 2000; 96: 271-276.

13. Ishihara N, Matsuo H, Murakoshi H, Laoag-Fernandez JB, Samoto $T$, Maruo $T$. Increased apoptosis in the syncytiotrophoblast in human term placentas complicated by either preeclampsia or intrauterine growth retardation. Am J Obstet Gynecol 2002; 186: 158-166.

14. Crocker IP, Cooper S, Ong SC, Baker PN. Differences in apoptotic susceptibility of cytotrophoblasts and syncytiotrophoblasts in normal pregnancy to those complicated with preeclampsia and intrauterine growth restriction. Am J Pathol 2003; 162: 637-643.

15. Guicciardi ME, Gores GJ. Life and death by death receptors. FASEB J 2009; 23: 1625-1637.

16. Schulze-Osthoff K, Ferrari D, Los M, Wesselborg S, Peter ME. Apoptosis signaling by death receptors. Eur J Biochem 1998; 254: 439-459.

17. Los M, Wesselborg S, Schulze-Osthoff K. The role of caspases in development, immunity, and apoptotic signal transduction: lessons from knockout mice. Immunity 1999; 10: 629-639.

18. Bantel H, Ruck P, Gregor M, Schulze-Osthoff K. Detection of elevated caspase activation and early apoptosis in liver diseases. Eur J Cell Biol 2001; 80: 230-239.

19. Hefler LA, Tempfer CB, Bancher-Todesca D, Schatten C, Husslein P, Heinze G et al. Placental expression and serum levels of cytokeratin-18 are increased in women with preeclampsia. J Soc Gynecol Investig 2001; 8: 169-173.

20. Bantel H, Lugering A, Heidemann J, Volkmann X, Poremba C, Strassburg CP et al. Detection of apoptotic caspase activation in sera from patients with chronic $\mathrm{HCV}$ infection is associated with fibrotic liver injury. Hepatology 2004; 40: 1078-1087.

21. Hamad RR, Bremme K, Kallner A, Sten-Linder M. Increased levels of an apoptotic product in the sera from women with pre-eclampsia. Scand J Clin Lab Invest 2009; 69: 204-208.

22. Seidel N, Volkmann X, Langer F, Flemming P, Manns MP, Schulze-Osthoff $K$ et al. The extent of liver steatosis in chronic hepatitis $\mathrm{C}$ virus infection is mirrored by caspase activity in serum. Hepatology 2005; 42: 113-120.

23. Joka D, Wahl K, Moeller S, Schlue J, Vaske B, Bahr MJ et al. Prospective biopsy-controlled evaluation of cell death biomarkers for prediction of liver fibrosis and nonalcoholic steatohepatitis. Hepatology 2012; 55: 455-465.

24. MacFarlane M, Merrison W, Dinsdale D, Cohen GM. Active caspases and cleaved cytokeratins are sequestered into cytoplasmic inclusions in TRAlL-induced apoptosis. J Cell Biol 2000; 148: 1239-1254.

25. Strand S, Strand D, Seufert R, Mann A, Lotz J, Blessing M et al. Placenta-derived CD95 ligand causes liver damage in hemolysis, elevated liver enzymes, and low platelet count syndrome. Gastroenterology 2004; 126: 849-858.

26. Prusac IK, Zekic Tomas S, Roje D. Apoptosis, proliferation and Fas ligand expression in placental trophoblast from pregnancies complicated by HELLP syndrome or pre-eclampsia. Acta Obstet Gynecol Scand 2011; 90: 1157-1163.

27. Eide IP, Isaksen CV, Salvesen KA, Langaas M, Gunther CC, Iversen AC et al. Fetal growth restriction is associated with reduced FasL expression by decidual cells. J Reprod Immunol 2007; 74: 7-14.

28. Cali U, Cavkaytar S, Sirvan L, Danisman N. Placental apoptosis in preeclampsia, intrauterine growth retardation, and HELLP syndrome: an immunohistochemical study with caspase-3 and bcl-2. Clin Exp Obstet Gynecol 2013; 40: 45-48.

29. Krueger A, Fas SC, Baumann S, Krammer PH. The role of CD95 in the regulation of peripheral T-cell apoptosis. Immunol Rev 2003; 193: 58-69.

30. Volkmann X, Fischer U, Bahr MJ, Ott M, Lehner F, MacFarlane M et al. Increased hepatotoxicity of tumor necrosis factor-related apoptosis-inducing ligand in diseased human liver. Hepatology 2007; 46: 1498-1508.

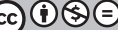

Cell Death and Disease is an open-access journal published by Nature Publishing Group. This work is licensed under a Creative Commons Attribution-NonCommercialNoDerivs 3.0 Unported License. To view a copy of this license, visit http://creativecommons.org/licenses/by-nc-nd/3.0/ 